\title{
Más allá del escombro. Causas de la ubicuidad, permanencia y visibilidad de paisajes ruinosos en las ciudades chinas
}

\section{Beyond Rubble. Causes of the Ubiquity, Permanence, and Visibility of Ruinous Urban Landscapes in China}

\author{
Xavier Ortells-Nicolau ${ }^{1}$
}

\begin{abstract}
RESUMEN
En las últimas décadas, como parte de la ingente urbanización en China, aparecen en el paisaje urbano parcelas derruidas y terrenos baldíos. Este artículo analiza las causas estructurales de la presencia y visibilidad de los espacios de demolición en función del análisis del conglomerado político, legal y económico de China.

Tras una breve descripción del desarrollo de las políticas urbanísticas durante el maoísmo, y de los cambios que conllevó el inicio de las reformas económicas, se desagregan el funcionamiento y actores principales del desarrollo urbanístico, se correlaciona la emergencia de la demolición con una serie de dinámicas, cadenas de autoridad y dependencias entre instituciones y agentes, y se establecen distintos tipos de espacios de demolición. Finalmente, se hacen notar reacciones y respuestas intelectuales y sociales a la demolición.
\end{abstract}

Palabras Clave: China; urbanismo; demolición; tipología; ruinas.

\footnotetext{
${ }^{1}$ Universitat de Barcelona, profesor asociado, Departamento de Lenguas y Literaturas Modernas y Estudios Ingleses; Grupo de Investigación ALTER, Universitat Oberta de Catalunya. xavier.ortells@ub.edu. ORCID iD: http://orcid.org/0000-0003-4834-4868. El autor quiere expresar su agradecimiento a Bret Wallach (greatmirror.com), Catherine Hyland y Mark Leong por las imágenes, a Pablo Martín Sánchez por la revisión y muy especialmente a los dos evaluadores anónimos por sus sugerencias, comentarios y atenta lectura.
} 


\begin{abstract}
Over the last decades, and as part of the vast programs of urbaniation in China, rubble, demolition, and wastelands have become widespread. This article analyzes the legal, political and economical framework to find structural causes for the permanence and visibility of said demolition.

After a succinct description of the development of urbanization under Maoism, and the changes brought upon by the economic reforms, the article unpacks the main actors in the function of urban development, it relates the emergence of demolition with a series of dynamics, chains of authority and dependences among institutions and agents, and establishes a typology of demolished spaces. Finally, it notes social and intellectual reactions to demolition.
\end{abstract}

KEY WorDS: China; urbanism; demolition; tipology; ruins.

Cómo Citar este artículo / Citation: Ortells-Nicolau, Xavier (2017): "Más allá del escombro. Causas de la ubicuidad, permanencia y visibilidad de paisajes ruinosos en las ciudades chinas", Estudios Geográficos, LXXVIII/282, pp. 317-338.

\title{
INTRODUCCIÓN
}

El espectacular crecimiento económico de China ha tenido uno de sus motores principales en el ingente proceso de urbanización llevado a cabo en las últimas décadas. Si bien China solo superó la barrera del 50\% de población urbana en el año 2011 (una fecha muy tardía en comparación a la mayoría de países occidentales, que sobrepasaron esta marca a principios del siglo Xx), en la actualidad la población urbana ya supone el 55\% (PRB, 2015) y se espera que continúe creciendo, alcanzando el $74,3 \%$ en 2045 (Naciones Unidas, 2015: 30). Entre 1980 y 2002, la época de mayor tasa de urbanización, la población urbana de China creció en 250 millones de personas (Hsing, 2010a: 5) - cerca de la población total de Indonesia, el cuarto planeta más poblado del planeta-, cifra a la que hay que añadir la población flotante de trabajadores migrantes sin registrar, que los últimos datos oficiales (2015) cifran alrededor de los 247 millones (Buró Nacional de Estadísticas).

La atracción que las ciudades han ejercido sobre la población rural, así como la recalificación administrativa de las ciudades ${ }^{2}$, han venido acompaña-

\footnotetext{
${ }^{2}$ La definición de ciudad en China difiere de la habitual en los países occidentales. El sistema urbano chino se establece alrededor de ciudades (shi o chengshi) y pueblos (zhen), y a menudo incluye zonas agrícolas periféricas (Zhang y Zhao, 1998). Desde el establecimiento de la República Popular (1949), la definición de ciudad ha cambiado en cuatro ocasiones (1955, 
das de un vertiginoso proceso de urbanización que, como destaca George Lin, no es un mero resultado del crecimiento económico sino un fuerza activa e instrumental para la transformación regional (Lin, 2011: 1). La magnitud de esa urbanización es apabullante. Como difundía el mismo Bill Gates, el historiador Vaclav Smil (2013) ha destacado que entre 2011 y 2013 China consumió más cemento (6,6 gigatoneladas) que los Estados Unidos en todo el siglo xx: solo en 2013, se urbanizaron 47.108 m² (Buró Nacional de Estadísticas).

\section{GRÁFICO 1}

INCREMENTO DEL PORCENTAJE DE POBLACIÓN URBANA A ESCALA NACIONAL, 1975-2013 (EXCLUYENDO LAS REGIONES ADMINISTRATIVAS ESPECIALES DE HONG KONG Y MACAO)

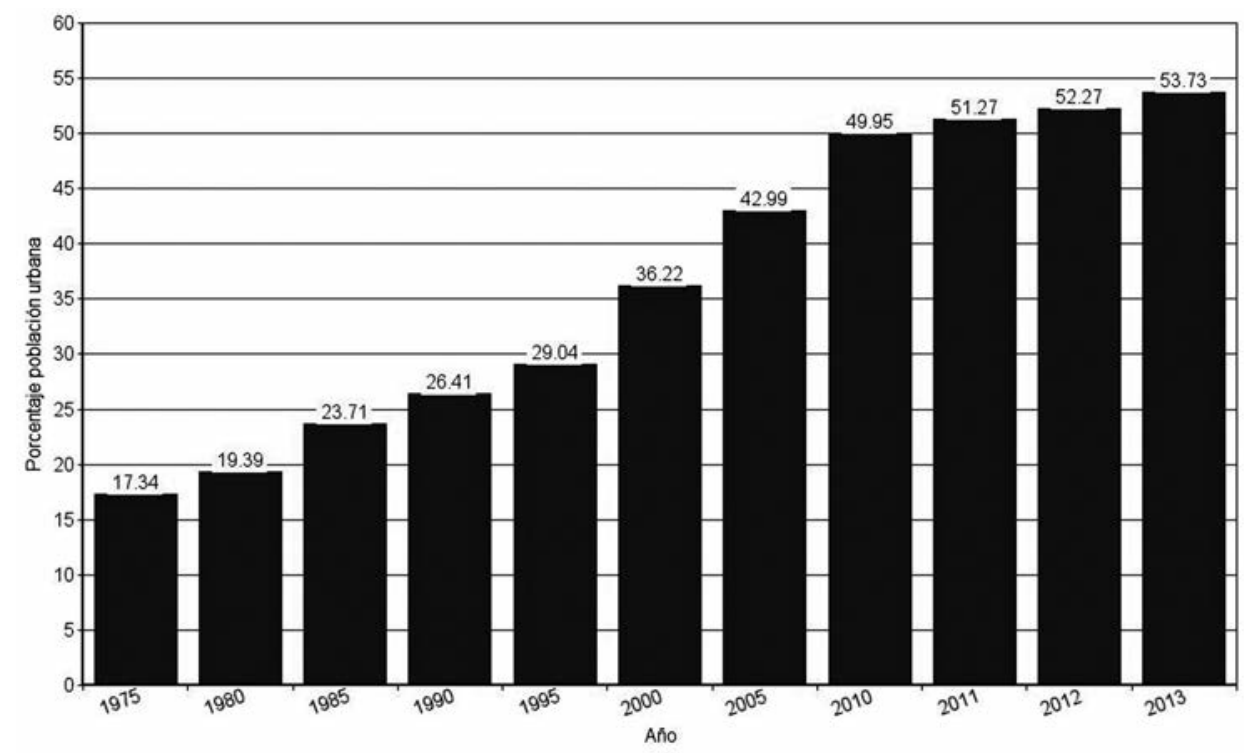

Fuente: Anuario Estadístico de China (Buró Nacional de Estadísticas).

1963/64, 1986 y 1993), lo que ha afectado al cómputo del crecimiento de ciudades y población urbana. Mientras que la definición de 1955 establecía las ciudades en virtud del tamaño de su población (a partir de 100.000 residentes), la de 1963 añadió a la definición la proporción de población dedicada a actividades no-agrícolas. En 1986 se redujo el criterio de población pero se añadió el del PIB y en 1993 se añadió la densidad de población como criterio (1.500 habitantes por $\mathrm{km}^{2}$ ) (Naciones Unidas, 2015: 77). A modo de ejemplo, el número de ciudades en 1978 era de 193, de 434 en 1988 y de 663 en el 2000 (Ren, 2013: 14). 
Además de una fuente de ingresos directos, la urbanización se ha erigido en estrategia de crecimiento y creación de valor, al aumentar los ingresos de sus ciudadanos e impulsar así el consumo doméstico. Junto a la economía, pues, la urbanización se plantea como plataforma para mejorar la «calidad» (suzhi) de los ciudadanos ${ }^{3}$ y adaptar la sociedad china a nuevas formas de interacción social y económica (Anagnost, 1997: 75-98).

La construcción de nuevas infraestructuras urbanas ha conllevado un proceso de expropiación, demolición y aplanamiento de viviendas y parcelas, y con ello, la recurrente aparición de paisajes de ruina y demolición en las ciudades chinas. En las obras de artistas y cineastas chinos, esta demolición ha condensado muchos de los cambios y desafíos de las reformas económicas, convirtiéndose en un icono clave de la China contemporánea ${ }^{4}$. Más allá del valor visual y/o estético de la ruina, como ha apuntando la geografía humana en los últimos años, las ruinas modernas son síntoma de dinámicas y conflictos sociales, procesos políticos y negociaciones de la hegemonía, del pasado, de la memoria, etcétera ${ }^{5}$. Por ello, y partiendo del trabajo de los estudios políticos y de urbanismo, este artículo explora el funcionamiento del sector inmobiliario chino así como sus estructuras legales, políticas y económicas. Al expandir el tratamiento del derribo más allá de una mera externalidad de la urbanización, se ponen en valor los procesos y los agentes políticos, económicos y sociales con el fin de localizar las causas de la emergencia y permanencia de la demolición urbana, así como su significado en el contexto social de la China contemporánea.

Primeramente, el artículo traza un breve recorrido por la evolución reciente del urbanismo en China desde el periodo maoísta (1949-1977) hasta la actualidad, centrándose en las dinámicas que animaron la emergencia del sec-

\footnotetext{
${ }^{3}$ En el contexto chino, el término suzhi se aplica habitualmente para referir el desarrollo (fazhan) educativo, cultural y económico de los ciudadanos. Dicha calidad no es innata y puede adquirirse, o mejorarse, por la vía de la formación y los cambios en hábitos de vida, siendo este el sentido que se apunta en el texto, dado que desde las instituciones responsables de la urbanización se entiende que la vida en la ciudad conlleva mejores opciones educativas, exige un mayor civismo y ofrece mejores oportunidades laborales (v. g., de cuello blanco). Ver Barmé y Goldkorn, 2013: 13.

${ }^{4}$ Entre los cineastas, cabe destacar el trabajo de Jia Zhangke, Zhang Yuan, Zhang Yang y Ning Ying y entre los artistas visuales, el trabajo del fotógrafo Rong Rong o los proyectos de Zhan Wang, Zhang Dali o Song Dong.

${ }^{5}$ Para una visión de conjunto de la revisión académica de la ruina, con especial énfasis en las propuestas de la geografía humana, ver Edensor y DeSilvey (2012). Para aportaciones de la geografía al estudio de la urbanización y la demolición en China, ver Hsing (2010a, 2010b) y Kao (2013).
} 
tor inmobiliario en el contexto de las reformas económicas iniciadas a finales de los años setenta, y pone de relieve las implicaciones del funcionamiento del sistema de propiedad dual del suelo. A continuación, se relacionan los resultados de este análisis con la aparición de distintos tipos de escenarios de demolición, así como con el contexto político, social e intelectual que enmarca la negociación del significado de la demolición y la ruina.

\section{Apuntes sobre el desarrollo urbano reciente en la República Popular de} CHINA

Pese al privilegio ideológico del campo sobre la ciudad durante el periodo maoísta, las ciudades siguieron ofreciendo mejores condiciones de vida, por lo que pese a descensos periódicos (de 0,16 puntos en la tasa anual de urbanización en el periodo comprendido entre 1966-1977; Naciones Unidas, 2013: 18) y una ralentización general del crecimiento (2,3 anual entre 1960 y 1970; Ren, 2013: 23), la población urbana siguió creciendo. Prueba del ímpetu de este crecimiento en los primeros años de la nueva República Popular fue el establecimiento, en 1957, del sistema de registro de residencia, o hukou, con el que se querían regular los flujos de ciudadanos rurales hacia las ciudades (un sistema, por otro lado, que se mantiene en la actualidad).

Beijing fue la ciudad que creció más continuadamente durante el periodo maoísta. De los 1,65 millones de habitantes de 1949, se pasaba, en 1990, a los 5,2 millones (Abramson, 2007: 47). Esto se debió, entre otros motivos, a la demanda de personal administrativo para la nueva capital y a acontecimientos puntuales, como el terremoto de 1976 en la vecina ciudad de Tangshan, que generó una oleada de desplazados a la ciudad. La escasez de viviendas, causada por una menguada inversión en urbanización, se resolvió a menudo de forma precaria, subdividiendo viviendas para acomodar otras unidades familiares, en lo que Hsing denomina la «socialización de la vivienda privada» (2010b: 19), una solución que contribuiría a la densificación y pauperización del parque de viviendas que más adelante justificarían los planes de renovación y desarrollo.

Tras la muerte de Mao Zedong en 1976 se fueron abandonando progresivamente sus políticas revolucionarias. El nuevo vicepresidente Deng Xiaoping, actuando de facto como nuevo líder del país, dio inicio al proceso de Reforma y Apertura (Gaige kaifang) y a las "Cuatro Modernizaciones» (Sige xiandaihua) con que se buscaba impulsar los sectores clave de la agricultura, la industria, la defensa nacional y la ciencia y tecnología. Este giro político hizo 
posible la inversión extranjera tras décadas de aislamiento e instituyó una tendencia hacia la descentralización política y fiscal, factores que allanarían el terreno para el desarrollo gradual del sector inmobiliario y urbanístico.

Las reformas empezaron a implementarse de forma gradual, tentativa y con límites geográficos precisos. El Sistema de Responsabilidad Familiar (Jiating lianchan chengbao zeren zhi), implementado de modo experimental en la provincia de Anhui a finales de los años setenta, permitía a las familias vender sus excedentes en un mercado de precios no regulados y constituyó un primer estadio en la gradual transición de una economía planificada a una de mercado. Por otra parte, la transformación de las empresas estatales en Empresas Rurales no Agrícolas (Xiangzhen qiye) permitió la capitalización de activos colectivos en unos emergente mercados inmobiliarios.

El impulso más decisivo a la urbanización fue la creación, a partir de 1982, de Zonas Económicas Especiales en las ciudades costeras de Shenzhen, Zhuhai, Shantou y Xiamen, un modelo que más adelante se expandiría a otras regiones y ciudades (catorce ciudades en 1984 y dieciocho en los años siguientes). Se iniciaba así una estrategia de crecimiento basada en la atracción de capital extranjero, que daría lugar a un desarrollo a distintas velocidades liderado por las ciudades ${ }^{6}$.

\section{Contexto del deSARrollo uRbanístico en El PERIOdo DE REFORMA}

Como parte de las reformas políticas, se concedió a los gobiernos locales una mayor autonomía, en lo que más que un retraimiento de la autoridad del estado supuso una actualización y rearticulación de su poder. En ese contexto, la planificación urbanística substituyó a la planificación económica de la época socialista como vehículo principal de la intervención del estado en las ciudades (Hsing, 2010a: 9), constituyendo una nueva forma diferente de intervención estatal, de menor escala espacial (Ma, 2005: 478). Si bien la introducción del Sistema de Responsabilidad Fiscal (Caizheng baogan zhi) suponía una disminución considerable de la asignación presupuestaria del gobierno central (del 80\% en 1976 a menos del 10\% en 1992; McGee et al., 2007: 17), se concedía simultáneamente a los gobiernos locales el poder de hacer efecti-

\footnotetext{
${ }^{6}$ «Las ciudades lideran los condados» (shi guan xian) ha sido desde entonces la estrategia administrativa y espacial que de facto ha transformado China en una economía dirigida por las ciudades, a través de la conversión de condados en ciudades (xian gai shi) o de la anexión de unidades suburbanas a las ciudades (che xian jian qu) (Ma, 2005: 486-490).
} 
vos sus derechos propietarios sobre la tierra y se les alentaba a explorar formas de movilizar capital para la inversión. Al establecerse el rendimiento económico como requisito para la promoción de los cuadros, se estimuló la competición (entre regiones, entre ciudades, así como entre diferentes distritos de ciudades grandes) por el aumento del PIB local, la tasa de industrialización y la atracción de inversión extranjera. El desarrollo urbanístico pasó a ser una de las principales estrategias para conseguir esos fines, ya que los gobiernos locales podían otorgar exenciones fiscales a empresas extranjeras durante los primeros años de implantación, o terrenos a bajo —o incluso a ningúncoste. La urbanización también se utilizó para crear las condiciones óptimas (de transporte, infraestructuras, etcétera) para la atracción de la inversión extranjera, una estrategia reflejada en distintas frases hechas, como «construir un nido atractivo para atraer al fénix» (zhuchao yinfeng) o «construir las carreteras para canalizar la fortuna» (lutong caitong) (Lin, 2011: 10).

Durante los primeros años de las reformas, los gobiernos locales obtuvieron beneficios ingentes. En un primer momento, el gobierno central había establecido un pago fijo de impuestos anuales pero en 1994 un nuevo sistema tributario obligó a los gobiernos locales a pagar impuestos en proporción a sus ingresos (Lin, 2011: 3). Dado que los beneficios derivados de las inversiones inmobiliarias no tenían que constar en los presupuestos y quedaban así exentas de tasación, estos cambios en política fiscal supusieron un impulso aun mayor a la urbanización. La recalificación de terrenos públicos para uso comercial en el sector inmobiliario y la expropiación de suelo rural en la periferia de las ciudades se convirtieron en las fuentes más importantes de generación de beneficios directos. Solo en el año 2010, los gobiernos municipales de Shanghai y Beijing obtuvieron cerca de 100 millones de yuanes (unos quince millones de euros) del arrendamiento de los derechos de explotación inmobiliaria, cantidad que no constó en sus presupuestos y que por lo tanto no fue gravada (Ren, 2013: 73).

A fin de aumentar el parque de viviendas (cuya escasez, desde la nueva perspectiva de mercado, había sido causada por la consideración de la vivienda como prestación durante el periodo socialista), se incrementó gradualmente el precio de los alquileres y se subvencionaron las ventas del parque de viviendas existente o de nueva creación (Zhou y Logan, 1996: 407). Otro de los motores principales del desarrollo urbanístico (y, de manera colateral, del inmobiliario) fueron los planes de renovación urbana. Planteados como herramienta para subsanar la antigüedad y el deterioro de las viviendas, estos planes se convirtieron rápidamente en plataformas para la expansión urbana (al compensar a los inquilinos desplazados con apartamentos en edificios de 
nueva construcción en barrios periféricos) y la especulación, así como una de las fuentes principales de beneficios para los gobiernos municipales.

Uno de los primeros planes de renovación que se implantó fue el Programa de Renovación de Viviendas Viejas y Deterioradas (Weijiufang Gaizao) de Beijing, iniciado en abril de 1990 y dirigido principalmente a las zonas residenciales del centro de la ciudad que conforman el entramado de callejones denominados hutong ${ }^{7}$. A lo largo de las últimas décadas, las viviendas de patio cuadrangular, o siheyuan $^{8}$, la tipología tradicional que estructuraba estos barrios residenciales, se habían masificado (a resultas de la anteriormente citada subdivisión de las viviendas) y deteriorado. En muchos casos, los patios se habían atestado de construcciones precarias, aumentando los problemas de higiene (que se sumaban a la frecuente ausencia de agua corriente o baños en las casas), las molestias entre vecinos y la falta de privacidad. Progresivamente, los originales siheyuan unifamiliares se convirtieron en dazayuan (patios abarrotados) ocupados por varias familias.

En preparación para el Programa de Renovación, el parque de viviendas de Beijing se dividió en cinco categorías: 1) construcciones modernas; 2) construcciones viejas en buen estado; 3) viviendas en buen estado estructural pero con techumbre, ventanas, puertas, tabiques, etcétera, en mal estado; 4) viviendas estructuralmente poco sólidas; y 5) viviendas peligrosas. Para ser considerada «deteriorada» (weijiu), más del 30\% de un área residencial debía pertenecer a los grupos 4 o 5, o más del $70 \%$ a los grupos 3, 4 y 5 . Al iniciarse el proyecto, seis millones cuadrados de suelo residencial de Beijing fueron declarados deteriorados (Abramson, 2007: 49), una afectación que creció al limitarse en 40.000 metros cuadrados el área mínima de intervención. En 1998, tras los primeros años de aplicación del programa, se habían demolido 4,2 millones de metros cuadrados de vivienda antigua (Fang y Zhang, 2003: 153).

El desarrollo del programa también afectó al valor de la propiedad, por lo que Fang y Zhang (2003: 149) no dudan en calificar este tipo de desarrollo urbanístico de operación especulativa a gran escala. Si bien el plan original incluía la reubicación de los inquilinos en nuevos distritos residenciales en la misma zona, una creciente centrifugación urbana fue desplazándolos a nuevos

\footnotetext{
${ }^{7}$ Los hutong se remontan a la dinastía Yuan (siglos XII-XIV), cuando pequeñas calles que convergían en un pozo fueron denominadas con la palabra mongol hottog (pozo).

${ }^{8}$ Junto al entramado de estrechos hutong, las diferentes alas de los siheyuan, distribuidas alrededor de un patio cuadrangular, protegían de las frecuentes tormentas de arena, ofrecían buen aislamiento y combinaban la organización del núcleo familiar extendido con una fuerte interrelación con el vecindario.
} 
suburbios residenciales, mientras el suelo céntrico anteriormente ocupado por usos residenciales se comercializaba a precios muy elevados en proyectos más rentables, como centros comerciales o de negocios, hoteles y viviendas de lujo (Campanella, 2008: 150).

\section{ESTRUCTURA Y FUNCIONAMIENTO DEL MERCADO INMOBILIARIO CHINO}

Pese a la magnitud de las reformas, la estructura socialista del Estado se mantuvo en su lugar. Ante la emergencia de un mercado inmobiliario, el Estado se aseguró la propiedad de la tierra estipulándola en la nueva Constitución de 1982, algo que curiosamente no había ocurrido en la anterior constitución maoísta. A fin de compaginar la titularidad estatal con el desarrollo del sector privado, en 1988 se establecieron una serie de medidas suplementarias a la Constitución. El artículo 10 sección 4 de la Constitución, que declara que ninguna organización o individuo podrá apoderarse, comprar, o vender terrenos o realizar otras transacciones ilegales de tierras, fue enmendado para hacer posible que el derecho al uso de la tierra pudiera ser transferido de acuerdo a las provisiones de la ley (Yeh, 2005: 52), lo que la Ley Administrativa del Suelo de ese mismo año vendría a regular. A partir de ese momento, los gobiernos locales podían arrendar, si no el suelo, sí los derechos de uso del suelo urbano (no así, en un principio, el rural) por una duración de setenta años para usos residenciales y cincuenta años para usos comerciales. En consecuencia, el sector inmobiliario chino se constituyó como un mercado de usos, no de propiedades.

Este sistema dual, donde la propiedad estatal del suelo coexiste con un mercado de arrendamiento, se tradujo en la creación de dos tipos de suelos: suelo primario y suelo secundario. El suelo primario es asignado (huabao) por el gobierno central a los municipios y agencias estatales (ejército, empresas estatales, universidades, etcétera). Estos actores pueden luego transferir (churang) los derechos de uso del suelo a actores privados o semi-privados en el mercado secundario, derechos que, a su vez, a menudo circulan a través de transferencias, alquileres, o son usados como colaterales de créditos (Lin y Ho, 2005). No obstante, antes de poder transferir los derechos de uso del suelo, los gobiernos municipales o de distrito tienen que preparar el suelo a través de empresas de desarrollo primario (frecuentemente, de titularidad mixta público-privada), en un proceso que se denomina como «cocinar» el suelo para que pase de «crudo» (primario) a «cocido» (secundario). «Cocinar el suelo», pues, implica distintas etapas: desde negociar el monto de las expro- 
piaciones y las condiciones de la reubicación de los inquilinos, hasta demoler, despejar y aplanar el terreno, así como, finalmente, proveer la parcela de las infraestructuras básicas y las conexiones a las redes de transporte, electricidad y agua ${ }^{9}$. Es precisamente durante este proceso de «cocción» cuando las dinámicas institucionales, la convergencia de intereses opuestos y la búsqueda de rentabilidad pueden provocar, como veremos más adelante, la demora, el estancamiento o incluso la interrupción de proyectos inmobiliarios y urbanísticos, dejando tras de sí las diferentes tipologías de paisajes y parcelas de ruina y demolición que han venido a saturar las ciudades chinas.

\section{Tipologías de la demolición}

A fin de dar cuenta de las causas de la aparición de diferentes tipos de espacios ruinosos, así como de su extendida duración, es necesario añadir a los incentivos y elementos estructurales del desarrollo urbanístico las dinámicas que se establecen entre actores participantes en el proceso de implementación de proyectos.

Pese a la percepción generalizada de uniformidad y control, el poder del Partido Comunista y el Gobierno no constituye un bloque monolítico. En un sistema definido como de «autoritarismo fragmentado» (Lieberthal y Oksenberg, 1988) existen pugnas internas y distintas cadenas de jerarquía y autoridad. Los ejes a través de los cuales tiene lugar esta negociación por el poder se denominan tiao y kuai. El eje tiao remite a la cadena de jerarquía vertical desde el centro a la periferia dentro de un mismo sector o función, por ejemplo, desde un ministerio hacia sus respectivas ramas provinciales, de condado o municipales. Por su parte, el eje kuai u horizontal describe dependencias y jerarquías en un mismo plano, como los que pueden darse entre dos ministerios o dos entidades locales. En el terreno inmobiliario y urbanístico, la negociación por la jerarquía a lo largo del kuai puede generar conflictos al más alto nivel, esto es, entre los diferentes ministerios con competencias en un mismo asunto, tal y como se da, en el desarrollo urbanístico, entre el de Tierra y Recursos (Guotu Ziyuanbu), el de Construcción y Desarrollo Urbano-Rural (Zhu-

\footnotetext{
${ }^{9} \mathrm{~A}$ este proceso se le denomina «aplanamiento y tres conexiones» (san tong yi ping), pero el nombre ha cambiado al aumentar las prestaciones: las «siete conexiones» incluyen, además de las ya citadas, el alcantarillado, las telecomunicaciones, el gas y los sistemas de calefacción; y las «nueve conexiones», la conexión a Internet y la separación de aguas residuales y pluviales (Chang, 2005).
} 
fang he Chengxiang jianshebu) y el de Cultura (Wenhuabu), este último en tanto supervisor de la Administración Estatal del Patrimonio Cultural (Guojia wenwu ju), encargada de la catalogación y preservación del patrimonio arquitectónico. Asimismo, en lo que atañe a las políticas urbanísticas puede existir competencia horizontal a nivel local entre las oficinas municipales del Buró del Suelo (Ziyuan ju) y el Buró de Reliquias Culturales (Wenwuguan). Por ello, la implementación de una política determinada depende en gran medida en cuál de las dos líneas de autoridad domina en un tema concreto, o cuál goza de una «relación de liderazgo» (lingdao guanxi) (Lieberthal, 2006: 186-188).

Sumadas a la compleja red de dependencias y autoridades fragmentadas a lo largo de estos dos ejes, existen también cadenas de autoridad más o menos difusas en las que agencias estatales o el mismo Partido Comunista tienen un gran papel. En el caso del desarrollo urbanístico, los intereses de agencias estatales como unidades (danwei) residenciales o industriales, el ejército, las empresas de propiedad estatal, universidades, hospitales, etcétera, poseedoras de grandes parcelas de terreno de alto valor en zonas céntricas de las ciudades - por lo que Hsing (2010a: 35-38) denomina a estos actores los «terratenientes socialistas» ${ }^{10}$ - entran a menudo en conflicto con los de las administraciones locales. Mientras que la autoridad de los municipios y distritos se vehicula, a través del tiao o eje vertical, con las autoridades superiores (provinciales, ministerios), en caso de conflicto de intereses con la autoridad local las agencias estatales pueden maniobrar para reclutar el apoyo de las burocracias de supervisión o usar contactos directos con las altas esferas del poder (v. gr., el Partido, el Politburó, instituciones financieras) (Hsing, 2006: 172).

\section{Tierras baldías}

Especialmente en el caso de autoridades e intereses contrapuestos, los planes de renovación urbana pueden servir a los gobiernos locales para afirmar y asentar su autoridad sobre parcelas de terreno. A través de procesos administrativos como la demarcación de áreas y la emisión de títulos de propiedad y de registros catastrales, los municipios alcanzan derechos propietarios sobre

${ }^{10}$ En un sintomático análisis de caso, Wang Hui (2008: 203-253) detalla las vicisitudes de una gran empresa que fue transformada de empresa estatal a empresa colectivizada en los años ochenta, para, más adelante, ser reclamada por el estado en un proceso en el que fue de crucial importancia los 253.000 metros cuadrados de terreno en el centro de la ciudad de los que disponía la empresa. 
parcelas específicas (Hsing, 2010a: 47-55) y, con ello, evitan o disminuyen el alcance de las disputas con terratenientes de menor legitimidad pero con poderosas conexiones políticas, como las empresas estatales o el Ejército.

En contraposición, durante el desarrollo de litigios y negociaciones administrativas, las partes interesadas pueden tratar de acelerar la resolución del caso, la obtención de la licencia o el beneplácito oficial por la fuerza de los hechos consumados (lo que en chino se conoce como xianzhan houzou, literalmente «decapitar primero e informar más tarde al emperador»). Así, parcelas céntricas han sido en ocasiones niveladas con anterioridad a la aprobación de un proyecto. Empresas constructoras primarias (las encargadas de «cocinar» la tierra y prepararla para la subasta de sus derechos de uso) capaces de absorber el riesgo inherente en anticiparse a la autorización oficial, pueden beneficiarse así, también, de la entrada de la parcela en mercados especulativos, y del aumento del valor del suelo a medida que el inicio de la construcción se va posponiendo. A consecuencia de estas dinámicas, las parcelas de terreno nivelado pueden permanecer como extensos terrenos baldíos durante largos períodos de tiempo a la espera de que se inicien los trabajos de construcción.

\section{Demolición progresiva}

Algo parecido ocurre con la aparición de un segundo tipo de paisaje ruinoso, el de la demolición progresiva. Hasta el año 2011, la regulación oficial permitía empezar los trabajos de demolición antes de que las negociaciones para la compensación y expropiación de los inquilinos hubieran concluido. Como resultado, las empresas constructoras y los promotores de inversiones inmobiliarias podían iniciar precozmente la demolición, para así minimizar las protestas y la oposición vecinal y tomar la delantera en la negociación de un proceso que a partir de ese momento se percibía como irreversible.

Otro factor importante en cuanto a la duración de los trabajos de demolición y del particular aspecto ruinoso de ciertas parcelas ha sido la emergencia de una economía derivada de la demolición, dedicada al reciclaje de material de construcción. El proceso de rescate de estos materiales, bien sea a cargo de los mismos propietarios de las casas (que en ocasiones reutilizarán el material en nuevas construcciones) bien de empresas dedicadas al reciclaje y posterior comercio con ladrillos, barras de acero o maderas en buen estado (de puertas, ventanas o vigas), requiere un lento desmantelamiento sin maquinaria pesada para evitar dañar los materiales, lo que requiere un trabajo intensivo con herramientas precarias (martillos, mazas). 
A esta economía paralela al desarrollo urbanístico hay que añadirle el rescate y reciclaje de materiales de construcción llevado a cabo por los llamados «grupos de asalto de construcción» (tuji jianfang). El interés de estos grupos, normalmente provenientes de zonas periféricas o de pequeños pueblos en proceso de ser anexionados por la imparable expansión de las ciudades, se halla en la reutilización de esos materiales para incrementar - a menudo de forma temporal, precaria e incluso peligrosa - el área construida de sus propias residencias. De este modo, los inquilinos consiguen negociar una mayor compensación en el momento de su desalojo, dado que esta se calcula en función del área construida de las viviendas (Kao, 2013: 41-42). El lento desmantelamiento y reciclaje llevado a cabo por todos estos actores genera una tipología del derribo que se asemeja al aspecto de las ruinas antiguas o al de las ciudades bombardeadas, con columnas sobreviviendo a techos y tabiques y montañas de escombros acumulados a la espera de su clasificación.

\section{FIGURA 1}

\section{DISTINTOS ESTADIOS DE DEMOLICIÓN Y CONSTRUCCIÓN. BEIJING}

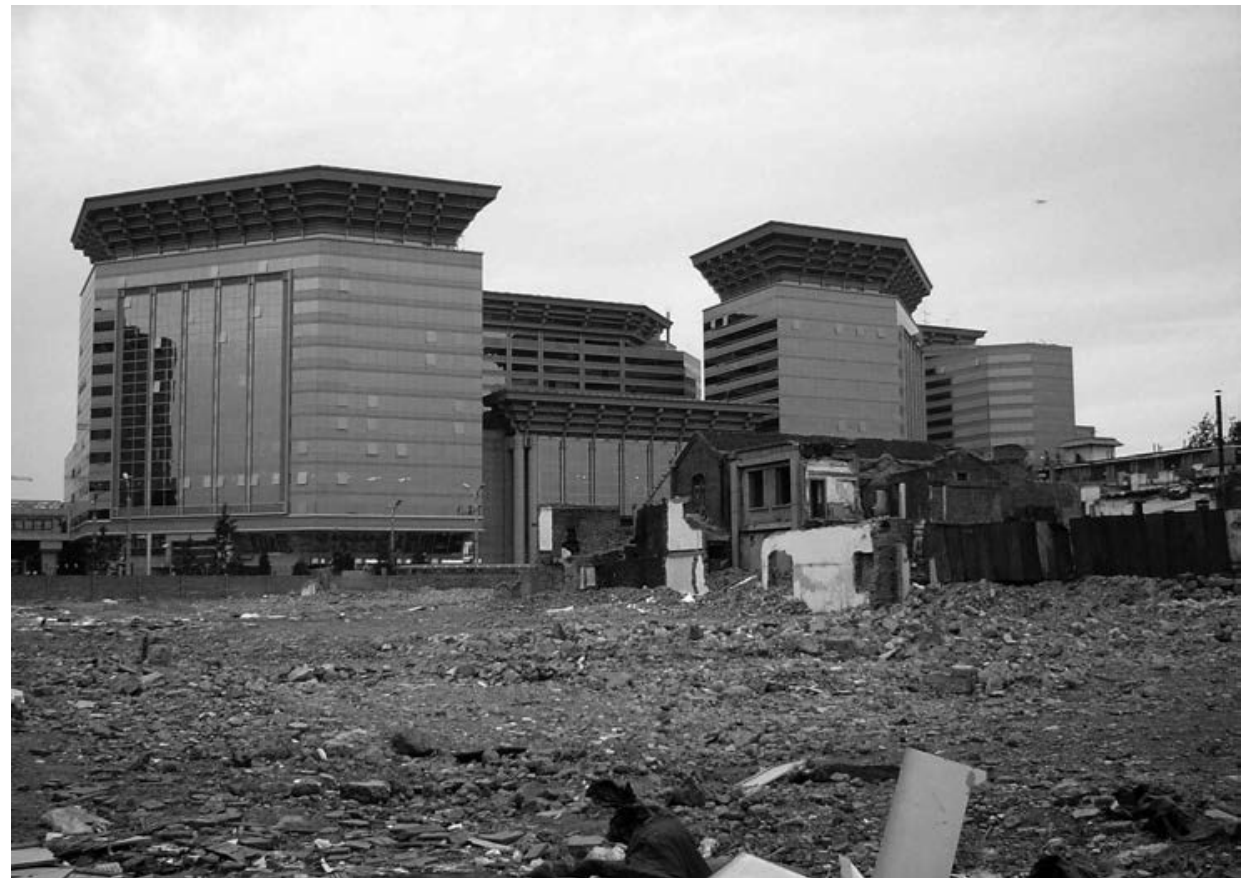

Fuente: greatmirror.com. 


\section{«Casas clavo»}

Los vecinos desplazados tienen frecuentemente escasas oportunidades o tiempo para presentar alegaciones o intervenir de manera efectiva y paritaria en las negociaciones que afectan a su compensación. Las quejas se centran en la insuficiencia del monto de la expropiación o en el tipo de propiedad, así como en la localización de la vivienda asignada, especialmente en el caso de propietarios de parcelas que incluían usos comerciales, como restaurantes o tiendas, y que son desplazados muy lejos de las zonas céntricas de alto valor comercial. Frente a las protestas, los promotores han llevado a cabo técnicas de intimidación y abuso sobre los inquilinos más resistentes, con casos documentados de agresiones e intimidación, destrozos de mobiliario o incluso de demolición de las viviendas con nocturnidad (Amnistía Internacional, 2012: 15-23; Hsing, 2010a: 77-78). Ha emergido así una nueva categoría de ciudadanos, los chaiqianhu o «inquilinos desalojados forzosamente», cuyas protestas han tomado en ocasiones un cariz dramático, como en el caso de Tang Fuzhen, una residente de la ciudad de Chengdu que se inmoló para tratar de impedir la demolición de su casa y cuyo caso motivó la revisión de las regulaciones sobre demolición (Li, 2009).

Algunos de los chaiqianhu, temiendo que en su ausencia sus propiedades fueran demolidas, han resistido parapetados en sus viviendas, progresivamente aisladas mientras la demolición avanzaba a su alrededor. Nacen así las llamadas «casas clavo» (dingzi hu), término basado en la comparación de esas casas con un clavo que se resiste a ser martilleado, que en algunos casos han conseguido una gran notoriedad pública.

\section{Lanweilou}

La precipitación con que muchos proyectos se inician y la improvisación inherente a un sector marcado por una gran indeterminación, también han afectado a proyectos inmobiliarios durante el periodo de desarrollo secundario, es decir, durante el proceso de construcción del inmueble o infraestructura. En este caso, el uso frecuente del suelo como colateral para créditos bancarios por parte de gobiernos municipales y empresas estatales (Walker y Buck, 2007: 57), la sobre-financiación o, por el contrario, la falta de suficiente apoyo financiero, o pleitos irresueltos sobre deudas e impagos pueden derivar en la interrupción de los proyectos. Como resultado, emerge un tipo particular de ruina, la de los proyectos inacabados que se mantienen en pie, en ocasiones durante años. En la lengua popular, se les llama lanweilou, término 
compuesto de lan («descompuesto», «podrido») y wei («cola»o «final»). Así, los 'edificios (lou) de terminación podrida' son aquellos cuya construcción se ha visto interrumpida o suspendida.

\section{FIGURA 2}

\section{LANWEILOU: EL PARQUE DE ATRACCIONES WONDERLAND}

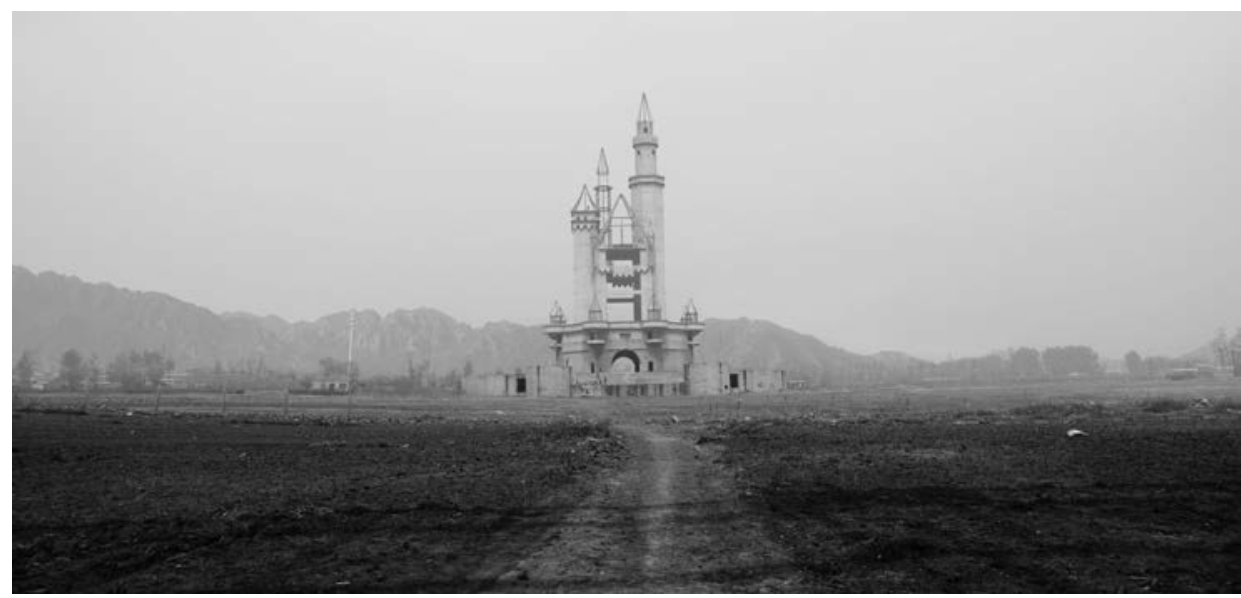

Fotografía de Catherine Hyland, parte del proyecto Wonderland (2011). Cortesía de la autora.

El fenómeno de los lanweilou tocó techo en los años noventa cuando, tan solo en la sureña isla de Hainan, proyectos con una extensión de 16,3 millones de metros cuadrados fueron abortados o inacabados. La audaz apuesta de Deng Xiaoping por el crecimiento económico y la generación de riqueza desató, a principios de los noventa, una fiebre desarrollista, con inversiones y precios que alcanzaron valores astronómicos al cabo de poco tiempo, una burbuja inmobiliaria que no soportó los intentos de controlar la creciente inflación con una mayor regulación del crédito y elevando las tasas de interés. Como resultado, se estima que proyectos de entre 30 y 50 millones de metros cuadrados de suelo edificado quedaron inacabados (Hsing, 2010a: 42). En otro contexto de incertidumbre económica tras la crisis asiática de $1997^{11}$, la construcción de las torres gemelas del complejo Zhongcheng Square en la ciudad

${ }^{11}$ No está de más notar que la abrupta explosión de la burbuja inmobiliaria española también ha dejado el territorio lleno de edificios y proyectos inmobiliarios sin terminar. La arquitecta Julia Schulz-Dornburg ha documentado este fenómeno en su proyecto Ruinas modernas. Una topografía del lucro (2012). 
de Guangzhou, que iban a convertirse en el edificio más alto de Asia, se interrumpió por problemas financieros, y no se terminó, tras una larga secuencia de litigios, hasta el año 2008 (Cao, 2015: 115-116), adquiriendo durante esa década el dudoso privilegio de ser «el lanweilou más grande de China» (Gaubatz, 2005: 102). Entre otros muchos famosos lanweilou, el parque de atracciones Wonderland, al noroeste de Beijing, ofrece una fascinante ruina de palacios de fantasía abandonados en medio del páramo que simboliza muchas de las aspiraciones y fracasos de la urbanización y el desarrollo chinos.

\section{Chai}

El carácter chino chai (拆) significa «demoler» y pintado en muros y paredes informa de la próxima demolición de una casa. Los barrios viejos de las ciudades chinas se han visto literalmente inundados de este símbolo que, pintado de manera tosca dentro de un precario círculo blanco sobre viejas pare-

\section{FIGURA 3}

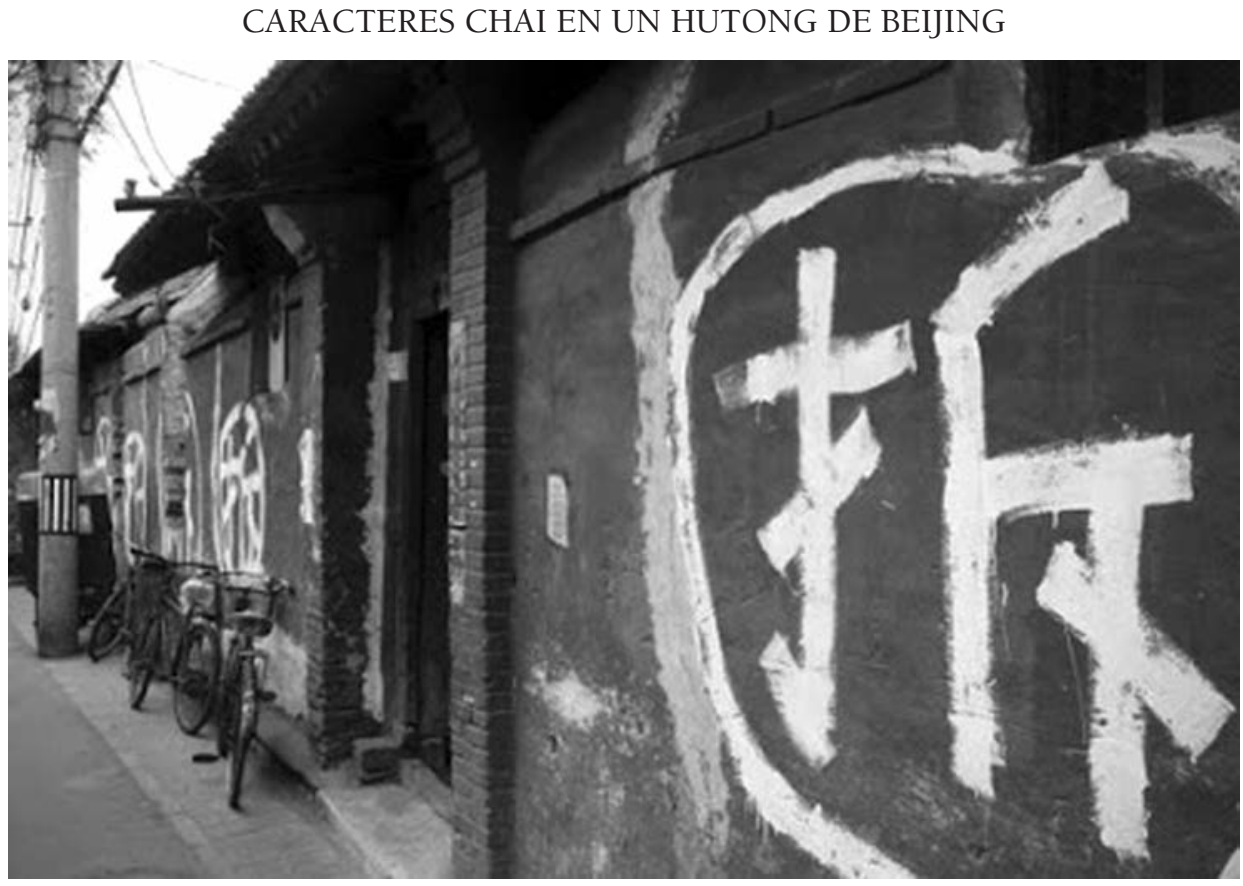

Fotografía de Mark Leong. Cortesía del autor.

Estudios Geográficos, Vol. LXXVIII, 282, pp. 317-338, enero-junio 2017

ISSN: 0014-1496, eISSN: 1988-8546, doi: 10.3989/estgeogr.201711 
des, adquiere algunos de los rasgos de la demolición: su improvisación, su rudeza, su inevitable visibilidad y su imposición; más allá de una nota informativa, el carácter chai devine una «escritura de poder» inscrita en las paredes que interpela a un tipo de sujeto y espera obtener comportamientos acomodaticios a la demolición (Chau, 2008: 195). El argot local ha hecho una creativa reapropiación del omnipresente carácter chai: China pasa a ser Chai-na, cuya pronunciación asemeja la del nombre del país pronunciado en inglés y que en sus caracteres chinos incluye el chai de «demolición» junto a un elemento fonético (na) usado para nombres extranjeros, con lo que se enfatiza la generación de chai / demolición a nivel nacional, así como la creciente participación de China en las dinámicas económicas y culturales de la anglobalización ${ }^{12}$.

\section{CONCLUSIONES}

La inclusión de la casa donde el célebre historiador de la arquitectura china, Liang Sicheng, y su primera esposa y colega Liu Huiyin, vivieron en los años treinta en Beijing, en un plan de renovación urbanística generó una significativa respuesta mediática, que tuvo el efecto de interrumpir la demolición de la casa y que la Administración Estatal para el Patrimonio Cultural (Guojia wenwu ju) declarara la casa un bien protegido. A pesar de ello, durante las vacaciones del Festival de Primavera del 2012 la casa fue totalmente derruida. En un gesto poco usual, la agencia de noticias estatal Xinhua publicó un editorial donde se lamentaba el derribo, se hacía hincapié en el hecho de que la casa había sido declarada «reliquia cultural inamovible» (buke yidong wenwu) y se hacía notar que la construcción de una réplica (opción recurrente en casos de destrucción del patrimonio, ofrecida como solución por agentes oficiales) no se adecuaba a los tres principios rectores de la preservación del patrimonio, esto es, que el objeto original (yuan wu) se mantuviera en su lugar original (yuan zhi) y en sus condiciones originales (yuan zhuang) (Tu, 2012).

Ejemplo paradigmático de la improvisación, adaptación y política de hechos consumados que rigen el desarrollo urbanístico, el caso de la casa de Liang y Lin también ilustra las jurisdicciones e intereses contrapuestos entre diferentes administraciones con competencias en el desarrollo y la gestión del

${ }^{12}$ El término anglobalisation, acuñado por el historiador Niall Ferguson para identificar el papel del Reino Unido en la integración a escala mundial de bienes de consumo, trabajo y capital a partir de 1850 (2003), ha pasado a connotar la fuerte impronta de los Estados Unidos y el Reino Unido en la globalización tras la Guerra Fría, una «globalización» mayormente «anglo». 
suelo y el patrimonio: de un lado, la Administración Estatal del Patrimonio Cultural y sus comités de ciudad y de distrito; del otro lado, el Buró de Recursos del Suelo, los oficiales del gobierno municipal y las empresas estatales, cuyos intereses coinciden a menudo con los de empresas privadas (en el caso de la casa de Liang Sicheng, como en muchos otros, se trataba de empresas de titularidad mixta público-privada; Branigan, 2012). Dado que en China existen políticas de preservación y conservación equivalentes, tanto en número como en rigor, a las de otros países occidentales, la iniciativa constructora y desarrollista de los gobiernos locales y de los inversores se suele señalar como el principal responsable de un desarrollo urbano implacable.

Asimismo, la respuesta frente a la amenaza de demolición de la casa — de relativo valor arquitectónico- arroja luz sobre las percepciones y valoración del patrimonio arquitectónico. El valor simbólico o histórico ha determinado, con más frecuencia que su valor arquitectónico o artístico, la preservación de algunos edificios emblemáticos. Si bien es cierto que la profesionalización del urbanismo desde los años noventa ha mejorado la valoración del patrimonio, el activismo a favor de la conservación arquitectónica se ha relacionado frecuentemente con áreas como la salud pública, la justicia social o la sostenibilidad ambiental y económica. No es de extrañar, entonces, que algunos de los mayores y más visibles promotores de la preservación arquitectónica, como los llamados «cuatro pilares de acero» (si da tiegan) de la preservación, sean prestigiosas personalidades públicas, hijos de importantes intelectuales e implicados en la lucha medioambiental ${ }^{13}$.

En el discurso oficial, las casas y barrios viejos son un obstáculo para una modernización que redunda en el bien colectivo. Sumado a los costes de renovación de las casas antiguas, imposibles de asumir para la mayoría de residentes (Martín Salvanyà, 2014: 190), los planes de reurbanización se plantean como una oportunidad para la expansión urbana, la mejora de las condiciones de vida y la modernización de la economía doméstica. Una vez derruidas las zonas residenciales en el centro, la elevación vertical de los nuevos edificios reemplaza un entramado horizontal de alta densidad, mientras que la búsqueda de rentabilidad del suelo centrifuga los usos residenciales hacia las afueras. A la vez, se pretende preservar el valor histórico y material del patrimonio arquitectónico con la construcción de réplicas (como en la céntrica ca-

${ }^{13}$ Liang Congjie (hijo de Liang Sicheng) y Yang Dongping son los fundadores de la primera ONG medioambiental de China, mientras que Li Yan y Shu Yi (el hijo del escritor Lao She) son miembros de la Conferencia Política Consultiva del Pueblo. 
lle Qianmen, al sur de la plaza de Tian'anmen, donde una placa atestigua el emplazamiento del original) y con una experiencia, mediatizada por el turismo, de la historia (como en los hutong de Beijing, donde se pasea a los turistas en ciclo-rickshaws y se les invita a conocer el interior de algunas casas). Como admite el activista Shu Yi, la gentrificación aparece a menudo como única vía posible para acomodar la preservación arquitectónica a las diferentes fuerzas económicas (Braester, 2010: 113-117), como en el complejo Xintiandi en Shanghai, un antiguo barrio shikumen de importante valor arquitectónico ${ }^{14}$ e histórico (pues allí tuvo lugar, en 1921, el primer congreso del recién fundado Partido Comunista Chino) que ha sido transformado en moderna zona comercial, de restauración y ocio. La resultante versión del patrimonio, esterilizada y gradualmente deshabitada, sirve de reclamo turístico y comercial, a la vez que articula una visión embellecida, cosificada y parcial de la historia y la cultura.

Entre un pasado urbanístico frecuentemente deteriorado y un futuro marcado por el desarrollo y la comercialización, la demolición emerge en las ciudades chinas con una realidad innegable. Enmarcados por la temporalidad y la incertidumbre, estos espacios son, en palabras del artista Ai Weiwei, un signo de interrogación, un inmenso vacío lleno de preguntas que sin embargo la gente prefiere ignorar (Blackwell, 2006: s. p.). La realidad material de la demolición, su alta visibilidad y duración, parecería cumplir funciones similares a las que Adam Chau apunta para el carácter chai, esto es, una cierta voluntad de «socializar» las reformas, de imponer su realidad por la fuerza de su ineludible realidad. La ausencia de cercados y vallas, la permanencia de montañas de cascotes y su coexistencia con la vida cotidiana de las ciudades chinas parecen sugerir una intencionada visualización de la demolición.

No obstante, el significado de la demolición es diverso y frecuentemente contradictorio. Si bien supone una violenta intervención en el entramado urbano, también es capaz de abrir un paisaje urbano saturado a las esperanzas individuales y colectivas. Más importante aún, como hemos tratado de demostrar en este artículo, la división de intereses y autoridades, la dispersión de la agencia y la gran gradualidad e improvisación inherente en el proceso, hacen difícil plantear la demolición como «representación». Por ello, más que ensayar una lectura de la demolición, este artículo ha querido señalar algunas de las causas estructurales que han dado lugar a la ubicua, duradera y altamente visible rea-

${ }^{14}$ Los shikumen son una tipología arquitectónica típica de Shanghai, de casas en hilera y construcción de ladrillo, que mezcla influencias de la arquitectura europea y china. 
lidad de la demolición. En concreto, se ha destacado la importancia de las estructuras institucionales, legales y económicas que han regulado el desarrollo urbanístico en la China de la reforma, la existencia de un sistema dual de propiedad estatal del suelo y de mercado de arriendo de derechos de uso, la competición entre distintas agencias y administraciones públicas, y la connivencia entre actores públicos y privados. Las dinámicas resultantes han favorecido el desarrollo apresurado, improvisado y especulativo de proyectos de renovación, así como su prolongación, discontinuidad o abandono, lo que ha desembocado en una duradera realidad de terrenos demolidos, aplanados y vacíos.

\section{BIBLIOGRAFÍA}

Abramson, Daniel (2007): "The Aesthetics of City-Scale Preservation Policy in Beijing", Planning Perspectives, 22/2, pp. 129-166.

Amnistía Internacional (2012): Standing Their Ground. Thousands Face Violent Eviction in China, Londres, Amnesty International.

Anagnost, Ann (1997): National Past-Times: Narrative, Representation, and Power in Modern China, Durham, Duke University Press, 240 pp.

Barmé, Geremie R. y Goldkorn, Jeremy (eds.) (2013): China Story Yearbook 2013. Civilising China, Canberra, Australian Centre on China in the World, ANU, 459 pp.

Blackwell, Adrian (2006): "Ai Weiwei: Fragments, Voids, Sections and Rings", Archinect, 21 de junio del 2006, http://archinect.com/features/article/47035/ai-weiwei-fragments-voids-sections-and-rings [Fecha de consulta: 9/4/2017].

Braester, Yomi (2010): Painting the City Red. Chinese Cinema and the Urban Contract, Durham, Duke University Press, 424 pp.

Branigan, Tania (2012): "Chinese Developers Demolish Home of Revered Architects", The Guardian, 30 de enero del 2012, http://www.theguardian.com/world/2012/ jan/30/chinese-developers-demolish-home-architect [Fecha de consulta: 9/4/ 2017].

Buró Nacional de Estadísticas de China, Datos anuales, http://www.stats.gov.cn/english/Statisticaldata/AnnualData/ [Fecha de consulta: 9/4/2017].

Campanella, Thomas H. (2008): The Concrete Dragon: China's Urban Revolution and What It Means for the World, Nueva York, Princeton Architectural Press, $336 \mathrm{pp}$.

Cao, Albert Junjian (2015): The Chinese Real Estate Market: Development, Regulation and Investment, Abingdon, Taylor and Francis, 406 pp.

Chang, Yung Ho (2005): "Learning from Uncertainty", AREA, 78, pp. 6-11.

Chau, Adam Yuet (2008): "An Awful Mark: Symbolic Violence and Urban Renewal in Reform-era China", Visual Studies, 23/3, pp. 195-210.

Edensor, Tim, y DeSilvey, Caitlin (2012): "Reckoning with Ruins", Progress in Human Geography, 37/4, pp. 463-483. 
Fang, Ke y Zhang, Yan (2003): "Plan and market mismatch: Urban redevelopment in Beijing during a period of transition", Asia Pacific Viewpoint, 44/ 2, pp. 149-162.

Ferguson, Niall (2003): Empire: How Britain Made the Modern World, Londres, Allen Lane, 417 pp.

Gaubatz, Piper (2005): "Globalization and the development of new central business districts in Beijing, Shanghai and Guangzhou”, en Laurence J. C. Ma y Fulong Wu (eds.), Restructuring the Chinese City. Changing society, economy and space, Londres y Nueva York, Routledge, pp. 109-123.

Hsing, You-tien (2010a): The Great Urban Transformation. Politics of Land and Property in China, Nueva York, Oxford University Press, 278 pp.

Hsing, You-tien (2010b): "Urban Housing Movilization", en You-tien Hsing y Ching Kwan Lee (eds.), Reclaiming Chinese society: the New Social Activism, Nueva York, Routledge, pp. 17-41.

Hsing, You-tien (2006): "Global Capital and Local Land in China's Urban Real Estate Development", en Fulong Wu (ed.), Globalisation and the Chinese City, Nueva York, Routledge, pp. 167-189.

Kao, Shih-yang (2013): The City Recycled: The Afterlives of Demolished Buildings in Post-war Beijing (tesis doctoral), Berkeley, University of California, 232 pp.

Li, Raymond (2009): "Demolition rules under scrutiny after self-immolation case", South China Morning Post, 9 de diciembre de 2009, http://www.scmp.com/article/700713/demolition-rules-under-scrutiny-after-self-immolation-case [Fecha de consulta: 9/4/2017].

Lieberthal, Kenneth (2006): Governing China. From Revolution through Reform, Nueva York y Londres, W. W. Norton \& Company, 528 pp.

Lieberthal, Kenneth y Oksenberg, Michel (1988): Policy Making in China. Leaders, Structures, and Processes, Oxford, Princeton University Press, $445 \mathrm{pp}$.

Lin, George C. S. (2011): "Territorialization of State Power through Land Development in Southern China", Cross-Currents: East Asian History and Culture Review, 1, http://cross-currents.berkeley.edu [Fecha de consulta: 9/4/2017].

Lin, George C. S. y Ho, Samuel P. S. (2005): "The State, Land System, and Land Development Processes in Contemporary China", Annals of the Association of American Geographers, 95/2, pp. 411-436.

Ma, Laurence J. C. (2005): "Urban administrative restructuring, changing scale relations and local economic development in China", Political Geography, 24/4, pp. 477-497.

Martín Salvanyà, M. (2014): "La nueva calle tradicional china: renovados y revalorados", Actas Encuentro de Jóvenes Investigadores en Geografía y Estudios del Territorio 2014 "La geografía ante el desafío global”, Baracelona, Universitat de Barcelona, pp. 189-192, http://issuu.com/ejig2014/docs/actas_ejig_2014/1 [Fecha de consulta: 9/4/2017].

McGee, T. G., et al. (2007): China's Urban Space: Development Under Market Socialism, Londres, Routledge, 249 pp. 
Naciones Unidas, Departamento de Asuntos Económicos y Sociales (2015): World Urbanization Prospects. 20014 Revision, Nueva York, Naciones Unidas, http://esa.un.org/unpd/wup/FinalReport/ [Fecha de consulta: 9/4/2017].

Naciones Unidas, Programa de Desarrollo (2013): China National Human Development Report 2013. Sustainable and Livable Cities: Toward Ecological Civilization, Beijing, China Translation and Publishing Corporation, http://hdr.undp.org/en/content/ sustainable-and-liveable-cities-toward-ecological-civilization [Fecha de consulta: 9/4/2017].

PRB, Population Reference Bureau (2015): World Population Data 2015, http://www.prb. org/ wpds/2015/ [Fecha de consulta: Octubre 2015].

Ren, Xuefei (2013): Urban China, Cambridge, MA., Polity Press, 218 pp.

Schulz-Dornburg, Julia (2012): Ruinas modernas. Una topografía del lucro, Barcelona, Ambit, 220 pp.

Smil, Vaclav (2013): Making the Modern World: Materials and Dematerialization, Chichester, West Sussex, John Wiley \& Sons, 242 pp.

Tu, Hongchang (2012): "Mingren guju zaohui shi chentong de wenhua zhi shang" [La demolición de la vieja casa de una personalidad es una triste pérdida cultural], Xinhua, 2 de febrero de 2012, http://news.xinhuanet.com/politics/2012-02/02/c_ 111480763.htm [Fecha de consulta: 9/4/2017].

Walker, Richard y Buck, Daniel (2007): "The Chinese Road. Cities in the Transition to Capitalism", New Left Review, 46, pp. 39-66.

Wang, Hui (2008): El nuevo orden de China. Sociedad, política y economía en transición, Barcelona, Edicions Bellaterra, 272 pp.

Yeh, Anthony Gar-On (2005): "Dual land market and internal spatial structure of Chinese cities", en Laurence J. C. Ma y Fulong Wu (eds.), Restructuring the Chinese City. Changing society, economy and space, Londres y Nueva York, Routledge, pp. 52-70.

Zhang, L. y Zhao, S. X. B. (1998): "Re-examining China's Urban Concept and the Level of Urbanisation", The China Quarterly, 154, pp. 330-381.

Zhou, Min y Logan, John R. (1996): "Market Transition and the Commodification of Housing in Urban China", International Journal of Urban and Regional Research, 20/3, pp. 400-421.

Fecha de recepción: 22 de abril de 2015.

Fecha de aceptación: 26 de noviembre de 2015. 